\title{
The role of game rules in architectural design environments
}

\author{
Pieter Pauwels, Ronald De Meyer, Maarten \\ Audenaert \\ Department of Architecture and Urban Planning \\ Ghent University \\ Ghent, Belgium \\ pipauwel.pauwels@ugent.be, first.last@ugent.be
}

\author{
Koen Samyn \\ Digital Arts \& Entertainment \\ HOWEST \\ Kortrijk, Belgium \\ firstname.lastname@howest.be
}

\begin{abstract}
Experimenting' and 'observing' are crucial actions in architectural design thinking. They rely heavily on the representation environment used (e.g. sketching, scale models, sketch tools, CAD tools, etc.) and the 'game rules' at play in these environments. In this brief paper, we study the role of this representation environment in the overall architectural design thinking process. From this brief study, we indicate two design and implementation approaches to implement and design with such game rules in virtual design environments.
\end{abstract}

Keywords-component architecture; CAD; design thinking; game engines; reasoning

\section{THE ROLE OF 'EXPERIMENT' AND ‘OBSERVATION’ IN ARCHITECTURAL DESIGN THINKING}

'Observation' plays a central role in many thinking processes. We would like to point to the long-standing and still ongoing research on reasoning processes, more specifically to the research by C.S. Peirce [1]. He indicates how people rely on a cyclic combination of abductive, inductive and deductive reasoning processes to make decisions. Design thinking research points in this direction as well when trying to clarify 'how designers think' [2]. This parallel between design thinking and the cycle of abduction deduction - induction can be summarised as follows.

When a designer 'synthesises the facts', for instance by preliminary sketches or physical models, he essentially creates an alternative observation of the same situation, which leads instinctively to abductive reasoning lines and thus to hypotheses about the design situation at hand. The 'continuous examples that come to mind from the architect's repertoire' indicate the importance of personal experiences of the designer in this abductive process. If a designer underwent 20 years of positive experiences with a grid layout to organise design situations, this has become a very strong and trustworthy rule within this designer's understanding of 'good architecture', and a higher probability value will consequently be attributed when making this hypothesis. By incorporating a hypothesis in a design, a designer consciously or unconsciously adds a whole set of rules to a design, rules that were attributed inductively to the added concepts throughout all kinds of personal experiences with this concept. By 'plugging in' these personal understandings or rule sets in a design, implications or predictions can be deduced. Based on these predictions, experiments are set up and gone through in each reasoning cycle, using a specific representation model. For instance, a designer may choose to just imagine the consequences of his hypothesis, he might actually make a sketch of the situation, or possibly build a detailed 3D representation. Whatever the designer chooses as a representation model, he will always make an observation of this experiment and make some conclusions inductively. Most often, this observation in itself is the starting point of a new reasoning cycle.

What we want to focus on in this paper, is the important role played by the elements 'experiment' and 'observation' in this thinking process, and how these relate to the usage of information and communication technology (ICT), more specifically of virtual worlds. By this investigation, we hope to understand the role that virtual worlds (can) play in an architectural design process, and subsequently to indicate how to further improve such virtual worlds.

\section{SUPPORT GIVEN BY VIRTUAL WORLDS}

Many types of virtual worlds are available for architectural designers. These include Computer Aided Design (CAD) tools, sketching tools, photo-editing software, game environments, etc. A recent overview on some of these design environments can be found in [3]. Considering a design process as outlined above, how do these environments support a designer's thinking? Basically all of them allow a designer to do experiments which are hardly feasible in a real world environment. Nobody can 'photo-edit' what he sees; it might be too costly to build an in-detail 3D scale model of an architectural design; etc. So basically, virtual design environments allow a designer to use a representation environment that he has no access to otherwise. Using this specific representation environment, he can conduct 'experiments', make 'observations' and finally inductively come to certain conclusions. Based on the observation and the final conclusions, new hypotheses emerge and a new cycle in the design thinking process starts $[1,2]$. Virtual worlds (CAD, sketch environments, game environments, etc.) can thus be considered additional representation environments alternative to existing 'traditional' representation environments (pencil sketches, paintings, physical scale models, etc.). 


\section{THE ROLE OF THE REPRESENTATION ENVIRONMENT}

When using a certain representation environment, one has to design within the boundaries of this representation environment: the rules of the game. Gravity, for example, is one of the most important game rules in a 'real world' representation environment. Any representation environment for physical scale modelling follows different rules, mainly because of the nature of material substitutes (e.g. glue instead of steel joints). The rules in such representation environments have always had a crucial influence on the eventual outcome of the design process. Simply by switching the representation environment, architectural designers might come to remarkable new insights, thereby shaping the further design thinking process.

Now that designers have a lot more alternative representation environments to choose from, the impact on the design process is further increasing. Several examples can be named of how a 2D CAD environment with a grid background is likely to produce a building with a rather orthogonal plan layout and with an often not so inspiring section design. Similarly, a representation environment with $3 \mathrm{D}$ geometry generation as one of its core game rules is likely to produce very complex three-dimensional shapes, simply because this is most often the outcome of complex mathematical formulae.

As has been outlined before, each of the available virtual worlds (and by extent also the traditional 'real world' representation environments) has its advantages and disadvantages, and none of them appears the 'best' environment to work in. One merely has to choose the environment one thinks best depending on the design questions at hand, and subsequently follow the game rules or switch representation environment.

\section{HOW DOES THIS AFFECT THE DESIGN AND} IMPLEMENTATION OF NEW REPRESENTATION ENVIRONMENTS

Considering the above and assuming that we are targeting the design and implementation of the nextgeneration virtual world for architectural design thinking support, what would be the required elements to incorporate in this virtual world?

One possibility is to design a virtual world that resembles the real world as closely as possible; a game in which the rules that are imposed on a designer in any real world situation are implemented as closely as possible. This not only includes gravity, but also more information-rich rules, such as weight-bearing capacities of a wooden beam, or the fact that you just cannot build a concrete shape in any imaginable freeform. This may lie within scope when relying on the functionality that we outlined in [4], which allows pulling any kind of information from the web for specific building elements (Fig. 1). However, this assumes that one is able to model all such rules exactly.

Another possibility might be to design and implement a virtual world that allows a conscious choice of the game rules, including, for instance, gravity, material-based behaviour, specific dimensions (4D, 3D, 2D, 1D), etc. This would be even more promising, as it might allow designers to switch the rules of the game while still in the game, thereby creating a maximum of flexibility. This approach is currently under research as a possible extension to our earlier work [4]. In this investigation, we rely on the Unity game engine and on semantic web technology. As the explicit description of behaviour through rules is an inherent part of semantic web technology, the above should be technically feasible.

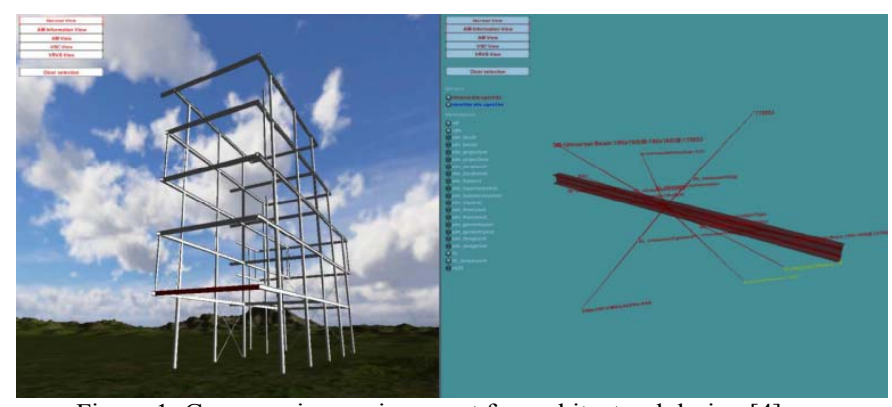

Figure 1. Game engine environment for architectural design [4].

\section{CONCLUSION}

We outlined how 'experimenting' and 'observing' are crucial elements in the (architectural) design thinking process. These actions typically rely on a specific representation environment, such as the real world, a $2 \mathrm{D}$ sketchy world, a CAD world, etc. One is required to follow a distinct set of 'rules of the game' when designing in such an environment (e.g. gravity, material properties, etc.). Such rules have a crucial impact on how design thinking progresses towards a final design. Choosing a representation environment is thus one of the most important choices to be made by the designer. We have outlined how virtual worlds, each with its own rules, has broadened the spectrum of available representation environments. Relying on game engine technology and semantic web technology, one might further be able to model even the set of rules itself in order to generate a design game of choice, furthermore allowing to design according to his/her own rules.

\section{ACKNOWLEDGMENT}

The authors gratefully acknowledge the funding support from the Research Foundation - Flanders (FWO).

\section{REFERENCES}

[1] C. S. Peirce, Collected Papers of Charles Sanders Peirce, vols. 1-6 (Eds. C. Hartshorne \& P. Weiss) (1931-1935), vols. 7-8 (Ed. A.W. Burks) (1958), Cambridge: Harvard University Press, 1958.

[2] E. Gardner, Reasoning in Architecture, Master thesis, TU Delft, 2009.

[3] A. Okeil, "Hybrid Design Environments: Immersive and NonImmersive Architectural Design," ITcon, vol. 15, pp. 202-216, March 2010.

[4] P. Pauwels, R. De Meyer, and J. Van Campenhout, "Visualisation of semantic architectural information within a game engine environment", Proceedings of the 10th International Conference on Construction Applications of Virtual Reality, 2010. 